\title{
Relativistic dust grains: a new subject of research with orbital fluorescence detectors
}

\section{B.A. Khrenov, N.N. Kalmykov, P.A. Klimov, ${ }^{*}$ S.A. Sharakin and M.Yu. Zotov}

Skobeltsyn Institute of Nuclear Physics, Lomonosov Moscow State University, Moscow 119991, Russia

E-mail: bkhrenov@yandex.ru, pavel.klimov@gmail.com

TUS (Tracking Ultraviolet Set-up) was the world's first orbital detector aimed at testing the principle of observing ultra-high energy cosmic rays (UHECRs) with a space-based fluorescence telescope. TUS was launched into orbit on 28th April 2016 as a part of the scientific payload of the Lomonosov satellite, and its mission continued for 1.5 years. During this time, its exposure reached $\sim 1550 \mathrm{~km}^{2} \mathrm{sr} \mathrm{yr}$ for primary energy $\gtrsim 400 \mathrm{EeV}$, and a number of extensive air showers-like events were registered. The shape and kinematics of the signal in these events closely resembled those expected from UHECRs but amplitudes of the signal and some other features were in contradiction with this assumption. A detailed analysis of one of EAS-like events (TUS161003) revealed that a primary cosmic ray would need to have an energy $\gtrsim 1 \mathrm{ZeV}$ in order to produce a light curve of the observed amplitude, which is incompatible with the cosmic ray spectrum obtained with ground-based experiments. More than this, the slant depth of the shower maximum be the signal produced by a cosmic particle, was estimated as $\lesssim 500 \mathrm{~g} / \mathrm{cm}^{2}$, which corresponds to cosmic rays around $1 \mathrm{PeV}$. We present a preliminary discussion of a hypothesis that the TUS161003 event and perhaps some other bright EAS-like events could be produced by relativistic dust grains, which were considered a possible component of the cosmic ray flux beyond the GZK cut-off some time ago.

$37^{\text {th }}$ International Cosmic Ray Conference (ICRC 2021)

July 12th - 23rd, 2021

Online - Berlin, Germany

\footnotetext{
${ }^{*}$ Presenter
} 


\section{The TUS detector}

A comprehensive description of the TUS telescope can be found in [1,2]. Here we will briefly outline its main features.

From the very beginning TUS, was designed as a pathfinder for a much more sophisticated KLYPVE experiment onboard the International Space Station [3, 4]. The main components of TUS were a Fresnel mirror and a square-shaped $16 \times 16$ photodetector aligned to the focal surface of the mirror. The mirror had an area of nearly $2 \mathrm{~m}^{2}$ and a $1.5 \mathrm{~m}$ focal distance. The field of view (FOV) of the telescope was $9^{\circ} \times 9^{\circ}$, which covered an area of approximately $80 \mathrm{~km} \times 80 \mathrm{~km}$ at sea level. Totally 256 Hamamatsu R1463 photomultiplier tubes (PMTs) were grouped in a $16 \times 16$ square and formed the focal surface of the photodetector. The FOV of each channel was around $5 \mathrm{~km} \times 5 \mathrm{~km}$ on ground. A glass UV filter was placed in front of every PMT to limit the measured wavelength to the 300-400 nm range. Light guides with square entrance apertures and circular outputs were used to uniformly fill the FOV. The high-voltage system was aimed to adjust the sensitivity of PMTs to the intensity of the incoming light and switch them off completely on day sides of the orbit.

The TUS electronics could operate in four modes with different time sampling windows. The main mode was intended for registering the fastest processes in the atmosphere and had the time step of $0.8 \mu$ s. Every record consisted of ADC codes written for all photodetector channels in 256 time steps with a total duration of $204.8 \mu$ s. We only discuss events registered in this mode of operation.

TUS was launched into orbit on 28 April 2016 as a part of the scientific payload of the Lomonosov satellite. The satellite had a sun-synchronous orbit with an inclination of $97.3^{\circ}$, a height about $470-515 \mathrm{~km}$ and a period of $\approx 94$ minutes. The TUS mission continued till 30 November 2017 , and the total exposure was estimated to be $\sim 1550 \mathrm{~km}^{2}$ sr yr at energies $\gtrsim 400 \mathrm{EeV}[5] .{ }^{1}$

\section{EAS-like event TUS161003}

The TUS161003 event was registered on 3 October 2016 in approximately $100 \mathrm{~km}$ south-east from Minneapolis, MN, USA [6]. The Richard J. Dorer Memorial Hardwood State Forest was in the field of view of hit pixels of TUS, and no potential sources of artificial UV light were identified on ground. The signal was registered in perfect observational conditions without any noticeable clouds except some small low-altitude ones, and low background illumination.

The light curve of the TUS161003 event and location of the hit channels in the focal surface are shown in Figure 1. The spatio-temporal dynamics of the signal found in 10 adjacent pixels was similar to what was expected from an extensive air shower (EAS) basing on detailed simulations performed with ESAF [7]. The arrival direction of the signal source was found to be $\theta=44^{\circ} \pm 4^{\circ}$, $\phi=50^{\circ} \pm 10^{\circ}$. However, a primary cosmic ray, be it a proton or an iron nucleus, would need an energy $\gtrsim 1 \mathrm{ZeV}=1000 \mathrm{EeV}$, which is at least three times higher than the most powerful cosmic ray event ever registered during more than 60 years of observations [8]. The limited exposure of the

\footnotetext{
${ }^{1} \mathrm{~A}$ high energy threshold in the estimation is partially due to a shortcut of the high-voltage system that took place on the first day of TUS operation. The accident resulted in 51 PMTs burnt out and a decrease of sensitivities of other channels.
} 
TUS mission and results on the energy spectra of the Pierre Auger Observatory and the Telescope Array [9] make registration of such an extreme energy event highly unlikely.

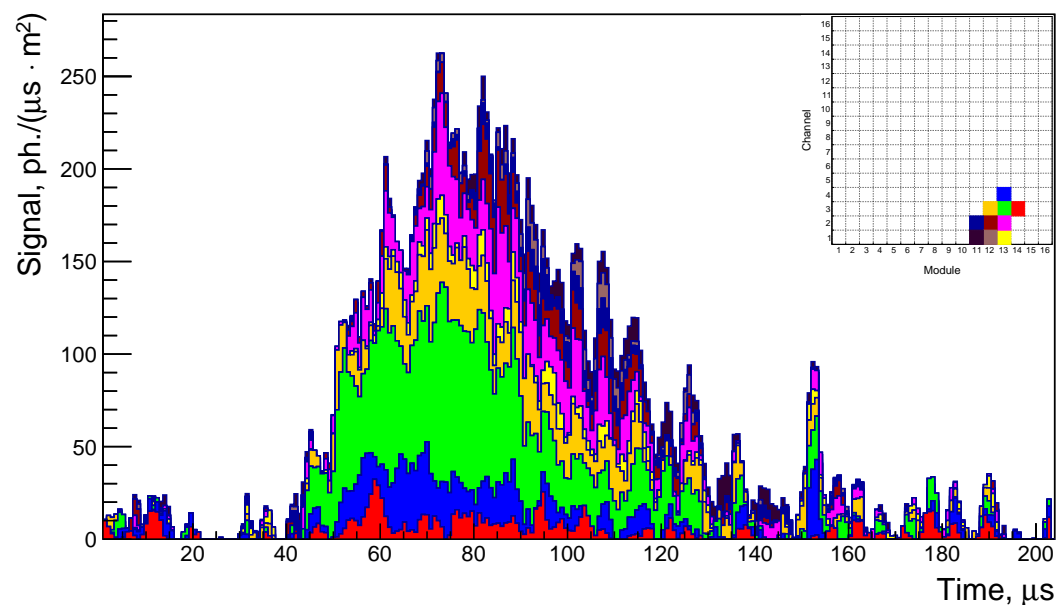

Figure 1: The light curve of the TUS161003 event and location of the hit channels in the focal surface of TUS [6].

Another argument against interpreting the TUS161003 event as an UHECR was the slant depth of the shower maximum. It was estimated from the light curve as $\lesssim 480-550 \mathrm{~g} / \mathrm{cm}^{2}$, which geometrically corresponds to altitudes $\sim 7.5-8.5 \mathrm{~km}$ above the ground. This rules out a proton or an iron origin of the primary source of light since a particle with an energy $\gtrsim 1 \mathrm{ZeV}$ arriving at the zenith angle $\sim 44^{\circ}$ should produce and EAS that hits the ground before reaching its maximum. Conversely, the observed slant depth corresponds to an EAS generated by a cosmic ray with an energy around PeV, see Figure 2.

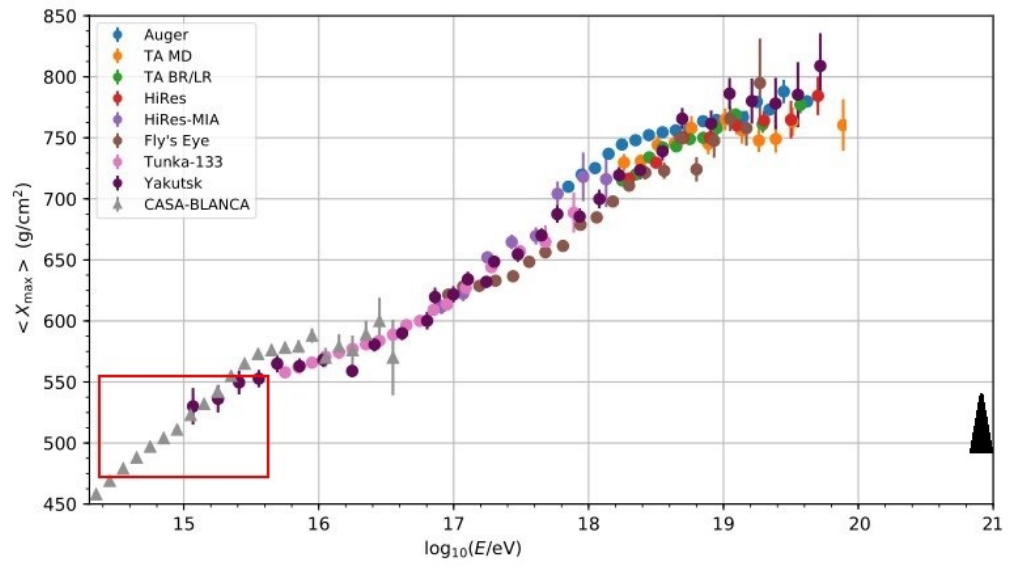

Figure 2: Mean depth of maximum of EASs vs. energy of primary cosmic rays according to data of different experiments. The figure is adapted from [10]. The red box shows the range of slant depths and respective energies estimated for the TUS161003 event. The black triangle shows estimations for the TUS161003 event. 


\section{Interpretation of TUS161003 as generated by a relativistic dust grain}

A number of artificial sources were considered in [6] in order to explain the TUS161003 event but only a peculiar configuration of ground flashers was found to be able to reproduce the kinematics and the light curve of the signal. As was pointed out in [6], a possible way to explain the TUS161003 event with an astrophysical phenomenon is provided by relativistic dust grains (RDGs). They were first considered by Spitzer [11] and later by Hayakawa [12] as possible sources of UHECRs of the highest energies. The idea was revisited in $[13,14]$ and [3] and a number of other studies. In particular, L. Anchordoqui performed detailed simulations of EASs produced by RDGs $[13,14]$ by means of the AIRES code ${ }^{2}$. The simulations were based on a number of assumptions:

- Relativistic dust grains encountering the atmosphere will produce a composite nuclear cascade.

- Each grain evaporates at an altitude of about $100 \mathrm{~km}$ and forms a shower of nuclei which in turn produces many small showers spreading over a radius of several tens of meters, whose superposition is observed as an extensive air shower.

- For the Lorentz factor $\Gamma \gg 1$, the internal forces between the atoms will be negligible.

- The nucleons in each incident nucleus will interact almost independently. Consequently, a shower produced by a dust grain containing $N$ nucleons may be simulated by the collection of $N$ nucleon showers, each with $1 / N^{\text {th }}$ of the grain energy.

Several sets of showers were generated in [13], each one for with different Lorentz factors. The samples were distributed in the energy range of $10^{18} \mathrm{eV}$ up to $10^{20} \mathrm{eV}$ and were equally spread in the interval of $0^{\circ}$ to $60^{\circ}$ zenith angle at the top of the atmosphere. All shower particles with energies above the following thresholds were tracked: $750 \mathrm{keV}$ for gammas, $900 \mathrm{keV}$ for electrons and positrons, $10 \mathrm{MeV}$ for muons, $60 \mathrm{MeV}$ for mesons and $120 \mathrm{MeV}$ for nucleons and nuclei. The particles were injected at the top of the atmosphere (100 km a.s.l), and the surface detector array was put beneath different atmospheric densities selected from the altitude of cosmic ray observatories (Fly's Eye, Yakutsk, AGASA, Auger). SIBYLL routines were used to generate hadronic interactions above $200 \mathrm{GeV}$. Results of these simulations were compared with experimental data, especially with an event recorded at the Yakutsk array on 7 May 1989 [15]. One of the conclusions of the work was that the dependence of the longitudinal profile of RDGs on the Lorentz factor is rather weak, and while RDG air showers must be regarded as highly speculative, they cannot be completely ruled out.

The study was continued in [14]. In this work, Anchordoqui et al. performed similar simulations but with a focus on heavy and "superheavy" nuclei. In particular, they studied longitudinal profiles of EAS generated by nuclei of different masses and RDGs. The particles were injected vertically at the top of the atmosphere, and the surface detector was put at sea level. The energy of the highest energy event, registered by Fly's Eye [8] was taken as a talon one. One of their results is shown in Figure 3. Notice that the shape of the profile of an EAS induced by a dust grain with the Lorentz

\footnotetext{
2http://aires.fisica.unlp.edu.ar/
} 
factor $\lg \Gamma=4.5$ is similar to the light curve of the TUS161003 shown in Figure 1, and its maximum is located at the slant depth $\approx 500 \mathrm{~g} / \mathrm{cm}^{2}$.

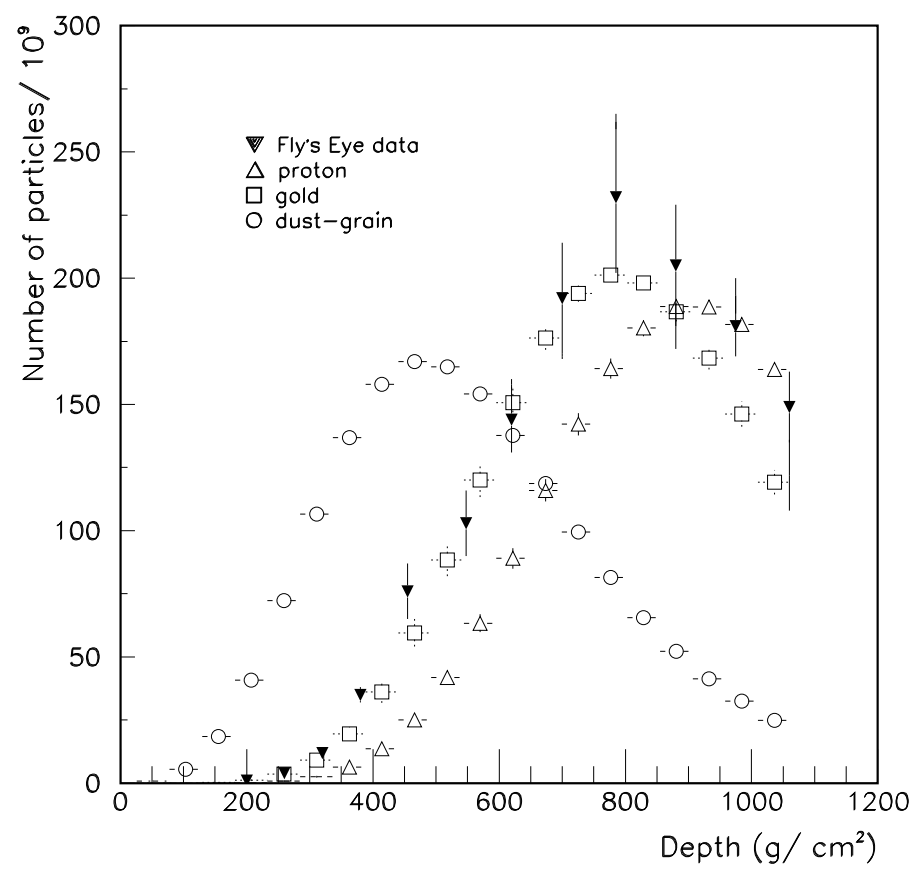

Figure 3: Longitudinal development of $300 \mathrm{EeV}$ showers generated by a proton, a gold nucleus and a relativistic dust grain with $\lg \Gamma=4.5$ together with the data of the highest energy CR, registered by Fly's Eys. Figure from [14].

One of the key ingredients of the studies by Anchordoqui et al. was the superposition model, which assumes that an average shower produced by a nucleus with energy $E$ and mass number $A$ is almost indistinguishable from a superposition of $A$ proton showers, each with energy $E / A$. The model is an approximate one but some experiments demonstrate that it might be sufficiently solid. In particular, a systematic study of meson multiplicity as a function of energy in nucleus-nucleus collisions was performed in [16], using cosmic-ray data in nuclear emulsion. It was shown that the data were consistent with the superposition model. The experiments gave identical results for final nucleons energy distribution in two cases: when the full history of primary nucleus fragmentation was taken into account, and when only division of a primary nucleus with an energy $E_{0}$ to $A$ nucleons was accounted. The superposition model had worked if the absorption mean free path in air of cluster fragments was much less than the absorption mean free path of cluster nucleons.

Suppose we consider the atmosphere to be a target for a cluster of nucleons (a dust grain) containing $N_{\mathrm{n}}=10^{6}$ nucleons in atomic and molecular states, with the primary energy of $E_{0} \approx$ $10^{21} \mathrm{eV}$ and energy per nucleon $E_{\mathrm{n}}=E_{0} / N_{\mathrm{n}}=10^{15} \mathrm{eV}$. Applying the superposition model to this impact process, one should expect as a final observable picture the sum of $N_{\mathrm{n}}$ EASs with the total energy approximately equal to $E_{0}$, excluding the bond energy summed over all nucleons of the cluster. For a silicon nuclei with the number of nucleons in one nucleus $A=28$, the bond energy per nucleon of about $\approx 8 \mathrm{MeV}$ and the number of nucleons-grain constituents $N_{\mathrm{n}}=10^{6}$, the energy spent on disintegration of all cluster constituents will be of the order of $10^{14} \mathrm{eV}$, which is negligible in comparison with an RDG projectile energy. 
In case of applying the superposition model to RDG primaries, it is important to keep in mind that molecules and atoms of RDG have very small absorption mean free path in air $\left(10^{-5}-10^{-4} \mathrm{~g} / \mathrm{cm}^{2}\right)$ in comparison with nuclear absorption mean free path, which equals to $14 \mathrm{~g} / \mathrm{cm}^{2}$ for iron and $25 \mathrm{~g} / \mathrm{cm}^{2}$ for silicon, known as the most abundant dust grains in non-relativistic range. This implies that the atomic type of a cluster impacting the atmosphere very soon (at altitudes of the order of a hundred kilometers) changes their original form to the sum of $N_{\mathrm{n}}$ independent nucleons.

\section{Discussion and Conclusions}

Orbital fluorescence telescopes aimed at observing UHECRs will provide an interesting opportunity for studying relativistic dust grains. While the remarkable coincidence of the slant depth of a shower maximum generated by an RDG shown in Figure 3 and that of the TUS161003 event does not necessarily mean that the signal registered by TUS could be produced by a dust grain, it suggests that this hypothesis is worth studying in detail. Following the superposition principle discussed above, one can check if an air shower generated by a relativistic dust grain that consists of $\sim 10^{6}$ protons with an energy around $1 \mathrm{PeV}$ is able to produce a light curve similar to that of the TUS161003 event or some other EAS-like events registered by TUS. Even in case it can, one should keep in mind that the superposition model is only an approximation, and more advanced models might be needed. Besides this, other studies argue that photoelectronic emission by optical and UV background radiation as well as Coulomb explosions due to collisional charging in interstellar and intergalactic medium play an important role in the destruction of relativistic dust grains in their way in space, which considerably reduces chances that they account for UHECRs [17]. This aspect should also be studied in details.

Nevertheless, taking into account that statistics of events beyond the GZK cut-off is very limited, and only a handful of UHECRs with energies above $100 \mathrm{EeV}$ have been registered [18], we believe one cannot completely rule out the possibility that a small fraction of cosmic rays of the highest energies is produced by relativistic dust grains. The Mini-EUSO telescope [19] that currently operates at the International Space Station and the future EUSO-SPB2 [20], K-EUSO [21] and the POEMMA [22] missions can extend the capabilities of TUS and the ground-based detectors and shed new light on this hypothesis. Meanwhile, we are going to study this possibility following the ideas outlined above and will report results elsewhere.

\section{Acknowledgments}

The work was supported by Space State Corporation ROSCOSMOS and by Lomonosov Moscow State University in frame of Interdisciplinary Scientific and Educational School of Moscow University "Fundamental and Applied Space Research." 


\section{References}

[1] P.A. Klimov, M.I. Panasyuk, B.A. Khrenov, G.K. Garipov, N.N. Kalmykov, V.L. Petrov et al., The TUS detector of extreme energy cosmic rays on board the Lomonosov satellite, Space Science Reviews 212 (2017) 1687 [1706. 04976].

[2] B.A. Khrenov, P.A. Klimov, M.I. Panasyuk, S.A. Sharakin, L.G. Tkachev, M.Y. Zotov et al., First results from the TUS orbital detector in the extensive air shower mode, Journal of Cosmology and Astroparticle Physics 9 (2017) 006 [1704.07704].

[3] B.A. Khrenov, M.I. Panasyuk, V.V. Alexandrov, D.I. Bugrov, A. Cordero, G.K. Garipov et al., Space program KOSMOTEPETL (project KLYPVE and TUS) for the study of extremely high energy cosmic rays, in Observing Ultrahigh Energy Cosmic Rays from Space and Earth, H. Salazar, L. Villasenor and A. Zepeda, eds., vol. 566 of American Institute of Physics Conference Series, pp. 57-75, May, 2001, DOI.

[4] V.V. Alexandrov, D.I. Bugrov, G.K. Garipov, V.M. Grebenyuk, M. Finger, B.A. Khrenov et al., Space experiment TUS for study of ultra high energy cosmic rays, International Cosmic Ray Conference 2 (2001) 831.

[5] F. Fenu, K. Shinozaki, M. Zotov, M. Bertaina and P. Klimov, Estimation of the exposure of the TUS space-based cosmic ray observatory, these proceedings, 2021.

[6] B.A. Khrenov, G.K. Garipov, M.A. Kaznacheeva, P.A. Klimov, M.I. Panasyuk, V.L. Petrov et al., An extensive-air-shower-like event registered with the TUS orbital detector, Journal of Cosmology and Astroparticle Physics 2020 (2020) 033 [1907 . 06028].

[7] C. Berat, S. Bottai, D. De Marco, S. Moreggia, D. Naumov, M. Pallavicini et al., Full simulation of space-based extensive air showers detectors with ESAF, Astroparticle Physics 33 (2010) 221 [0907 . 5275].

[8] D.J. Bird, S.C. Corbato, H.Y. Dai, J.W. Elbert, K.D. Green, M.A. Huang et al., Detection of a Cosmic Ray with Measured Energy Well beyond the Expected Spectral Cutoff due to Cosmic Microwave Radiation, Astrophys. J. 441 (1995) 144 [astro-ph/9410067].

[9] O. Deligny, The energy spectrum of ultra-high energy cosmic rays measured at the Pierre Auger Observatory and at the Telescope Array, in 36th International Cosmic Ray Conference (ICRC2019), vol. 36 of International Cosmic Ray Conference, p. 234, July, 2019.

[10] Telescope ArRay collaboration, Depth of Ultra High Energy Cosmic Ray Induced Air Shower Maxima Measured by the Telescope Array Black Rock and Long Ridge FADC Fluorescence Detectors and Surface Array in Hybrid Mode, Astrophys. J. 858 (2018) 76 [1801.09784].

[11] L. Spitzer, On the origin of heavy cosmic-ray particles, Phys. Rev. 76 (1949) 583.

[12] S. Hayakawa, Dust grain origin of cosmic ray air showers, Astrophysics and Space Science 16 (1972) 238. 
[13] L.A. Anchordoqui, Cosmic dust grains strike again, Phys. Rev. D 61 (2000) 087302.

[14] L.A. Anchordoqui, M.T. Dova, T.P. McCauley, T. Paul, S. Reucroft and J.D. Swain, A pot of gold at the end of the cosmic "raynbow"?, Nuclear Physics B Proceedings Supplements 97 (2001) 203 [astro-ph/0006071].

[15] N.N. Efimov et al., Peculiarities of Muon Component in Giant EAS, in Astrophysical Aspects of the Most Energetic Cosmic Rays, M. Nagano and F. Takahara, eds., p. 434, Jan., 1991.

[16] T.W. Atwater and P.S. Freier, Meson multiplicity versus energy in relativistic nucleus-nucleus collisions, Phys. Rev. Lett. 56 (1986) 1350.

[17] T. Hoang, A. Lazarian and R. Schlickeiser, On Origin and Destruction of Relativistic Dust and its Implication for Ultrahigh Energy Cosmic Rays, Astrophysical Journal 806 (2015) 255 [1412.0578].

[18] Pierre Auger collaboration, Measurement of the cosmic-ray energy spectrum above $2.5 \times 10^{18}$ eV using the Pierre Auger Observatory, Phys. Rev. D 102 (2020) 062005 [2008.06486].

[19] S. Bacholle, P. Barrillon, M. Battisti, A. Belov, M. Bertaina, F. Bisconti et al., Mini-EUSO mission to study earth UV emissions on board the ISS, The Astrophysical Journal Supplement Series 253 (2021) 36 [2010 . 01937].

[20] J. Adams, James H., L.A. Anchordoqui, J.A. Apple, M.E. Bertaina, M.J. Christl, F. Fenu et al., White paper on EUSO-SPB2, [1703.04513].

[21] F. Fenu, S. Sharakin, M. Zotov, N. Sakaki et al., A performance study of the K-EUSO space-based observatory, these proceedings, 2021.

[22] POEMMA collaboration, The POEMMA (Probe of Extreme Multi-Messenger Astrophysics) observatory, JCAP 06 (2021) 007 [2012 . 07945]. 